\title{
SCENARIOS PEDAGOGICAL-EDUCATIONAL MOVEMENT PLAY
}

\author{
Francesco Perrotta
}

Angelo Pannelli, Department of Education, University of Mcerata, Italy

Received 2010-10-19; Revised 2013-01-01; Accepted 2014-01-29

\begin{abstract}
The playtime and recreation plays an important role in child development. "Surplus energy" Spencer says That Both the man and the animal have the excess energy That are used in the activity fun. As we descended into the educational development Spencer Noted there was a decrease of fun Because The energy was used to meet basic needs. He Noted That in the higher animals there is a greater conservation of energy That Is Expressed in the activity fun. In 1900 Carl Cross argued That the recreational activity is a kind of exercise used to develop the individual's mental and motor activities. A sort of pre-exercise To ensure that structures are innate Certain transformed into complex structures and more December Especially Those Which are most suitable to environmental changes. The recreational activities are growing and changing together with the child's psychological and intellectual development, even if they REMAIN a milestone in the life of every person regardless of his age. Through the game as Schiller reminds us, "the man is fully human Only When he plays", through the game as everyone keeps his mind free from any thought, I know it has to be atto download his emotions and his instinct. The Game Becomes meaningful to the child's intellectual development, as When he plays, he Managed to surprise Himself by surprise and acquires new ways That allow them to interact with the outside world. In the game, the child Develops Their intellectual capabilities, emotional and relational. Becomes an instrument for the child Because it helps to develop creativity, helps him to experience cognitive abilities, so he can get in touch with his peers, gives life to the development of his personality.
\end{abstract}

Keywords: Sports Pedagogy, Play Activities, The Child's Intellectual Development, Development of Creativity, The Development of his Personality, Enhancement Areas of Intellectual, Emotional and Relational

\section{INTRODUCTION}

The recreational activity and recreation plays a very important role in the development of the child. "Surplus energy" Spencer maintains that both the man and the animal have the excess energy that are used in the activity fun. As we descended in the educational development Spencer noticed that there was a decrease of the play because the energy was used to meet basic needs. He noted that in the higher animals there is a greater conservation of energy is expressed in the activity fun. In 1900 Carl Cross argued that the recreational activity is a kind of exercise used to develop motor skills and mental health of the individual. A sort of pre-exercise to make sure that certain innate structures are transformed into more complex structures and more particularly suitable for those which are environmental changes.

Through the game, the child begins to understand the functioning of the objects. There is talk of game functional, although it is not a true recreational activity but an exercise, an imitative activity compared to real life situations. We start talking about real playful activity when the game begins to acquire the first functional representative characters, that the child uses functional objects, in this case we are talking about Game representative. The experience of the game teaches the child to be persistent and to have confidence in their 
abilities, it is a process by which it becomes aware of his inner world and the outer world, beginning to accept its legitimate needs of these two realities (Zavalloni, 1979; Bertolini, 1996; Solomon, 1999; Demetrius, 1992; 1996; Demetrio, 1990; 1995; Joints, 1983; Dewey; 1974; 1977; 1984; Castelnuovo, 2004; Mucchielli, 1987; Leo and Prezza, 2003; Mattelart, 1997; Pati, 1984; Cannao, 1989; Morselli, 1989; Contini, 1992; Frank, 1974).

Recreational activities grow and change to keep pace with the child's psychological and intellectual development, even if they remain a milestone in the life of every person regardless of his age. Through the game reminds us that Schiller, "man is only fully human when he plays', because through the game everyone keeps their mind free from any thought, it has no way of being able to download his emotions and his instinct.

The game becomes significant for the intellectual development of the child, as when he plays, he manages to surprise himself by surprise and acquires new ways that allow it to interact with the outside world. In the game, the child develops their potential intellectual, emotional and relational.

Becomes an instrument for the child because it helps to develop creativity, helps to experience cognitive abilities, he was able to enter into a relationship with his peers, gives life to the development of his personality.

\section{THE GAME: DEVELOPING EMOTIONAL, COGNITIVE AND SOCIAL}

During sensory-motor, which covers the first two years of life of the individual, the game is the main activity for his cognitive development and imitation, which begins as an imitation of sounds. The game is initially operating play.

Piaget argues that the progressive adaptation of the child to the environment, the game would occur every time, having acquired a skill or had made a discovery, the child tries to join the scheme motor or cognitive just bought new objects. In addition, the child learns to assimilation and accommodation. Knowledge is therefore based on the interaction between subject and object in the sense that the person acting on the object comes to editing.

To know is to assimilate reality and then "accommodate you" in thought patterns becoming more complex. The different game modes, depend on the child's emotional development and tend to change with growth. This stage becomes a key to reading his mental balance. The various stages can be listed as follows:
0-1 years: The play activity begins in the first months of a child's life.

Initially this first phase guarantees the child the feelings that go to reward and enrich one's self that is forming. The first games are made with your own body and that of the mother, even if his attention is also paid to the objects that surround it. The child shaking hands, moves his legs.

These are all activities that are purely exploratory and repetitive. The actions would follow one another and serve to ensure that you learn to distinguish between self and not-self:

2 years: At this stage of its development the child begins to become aware of the separation from the mother and therefore must cope with the crises of anxiety and abandonment. At this stage gives way to the transactional object. It 'an object that in the first years of life takes on a particular character, is offered to the child from the main figure of occurrence of the child (the mother) and when the mother is absent, this object represents and is a sign of certainty. Later, when the child acquires the knowledge of the mother figure and the transactional object is abandoned, it remains becomes a usual form, it loses its main meaning.

3 years: At this stage of growth begin to pass the first games of socialization. The child shows interest in playing with others. Begins to develop imaginative capacity, we tend to imitate the behavior of others.

4-5 years: It 'a stage where the game becomes an expression of their internal dynamics. games are the most favorite doll, the doctor, the game of hide and seek. The use of these games are used to represent the punishments and prohibitions that the child suffered.

6-10 years: The games are characterized by the rules and take place in the group. This means that the child learns to be with others and respect for the rules to ensure the smooth running of the game.

The importance of the teaching of sport and physical activity at school implies a set of knowledge and pedagogical skills. A pedagogy of sport can be defined as a set of procedures and techniques of learning, but that can only be understood in relation to goals or standards that are assigned to serve the purposes of the school. 
As with every subject taught in school, can be advanced one goal: That of relative mastery of the tasks that constitute it. Knowing how to count, how to swim practices are considered useful and necessary to our social life and from this point of view, their teaching is a part of its legitimacy. Improve access for all to the practice of one or several sports is part of this perspective. In this case we speak of sports culture. However, if the sport is taught in school, not only because everyone can practice it, but also because it allows you to develop a set of skills necessary to take appropriate motor conducted, as required by our social life today and tomorrow.

In addition to a sports culture, the goal is a culture of the body as a share of global education that hopes to overcome the mere transmission of adaptation techniques to promote the possibilities of evolution and creation. In this sense we can say that the physical and sporting activities are simultaneously subject to a quality education and as a means of physical and sports. This dual perspective that links utility and concern for the development of the individual, marks the two poles of all educational activity. During the last century pedagogues of sport and physical stress the difficulty in properly tie these two intentions.

At the cognitive level the game favors the development of memory, attention, aids concentration, the ability to compare, to relate, to use the perceptual patterns. Poor and inadequate recreational activity may contribute to deficiencies in cognitive level.

At the social level the game is manifested in three stages: Solitaire game, parallel and social.

The solitaire game is typical in children a few months old. It is characterized by the lack of social interaction. The game parallel appears between the first and third year of life. At this stage there is a moment of mutual help even if it comes to individual game.

The social game, typical of children aged between four and five years of life, is the beginning of the school term and is characterized by greater social interaction.

The playful activity acquires a great importance for understanding the evolutionary development. The evaluation of the game is important because it is through the systematic sequences and then ordered. These phases correspond to as many stages of a cognitive nature, so evaluate the development of the child's play allows you to assess cognitive functioning. The game then plays a dual role in the evolutionary development:

- Allows the child to understand the reality external to him (from which the world is still not included) and allows a good fit
- Allows the child to learn, play and control your inner world made of desires, impulses, instincts and then create the right compromise between the two

Through play, the child may know, understand and internalize order each new experience and acquisition. E 'can interpret their wishes and give them some form of design. E 'can feel the harmony and not conflict and contradiction often related to the presence of two worlds:

- The inner and

- External reality

With the game you have the ability to continuously acquire new cognitive skills, through the exploration of objects, active experimentation related to the multiple use of objects and the laws governing their interactions.

The recreational activity is considered important from the point of view of socialization, not only from the point of view of socio-emotional, but as a tool that allows the child to know, to control and manage the frustrations that are solicited from social life, from relationships with others and therefore understand their subjective needs and mediate with those of others.

\section{THE GAME IN SEVERAL EXPERTS}

The game is a human activity that generates satisfaction and pleasure. In fact, everyone chooses his game according to their own interests and their own pleasure. The recreational activity is the form of expression by the child form through which he refers to himself and explore the world around them. The game has the characteristic of being oriented towards creativity is in fact in the play he is having the opportunity to intervene actively on the elements that surround it. The dynamic that takes place in the game, is that it enables the child to gain self-awareness, to internalize norms, values and social roles.

Are cited below the great educators who contributed to the study of cognitive-motor development.

Froebel says that the game is the activity that characterizes the child up to childhood: It is through play that he internalizes the external world. Education must satisfy the spontaneous human activity without proposing any external models to imitate, but by providing the opportunity for each individual to realize their own. The game reveals the most intimate trends of each and the child interprets it as entertainment but as a real job. For this purpose, designed the kindergartens, ie spaces equipped for the game and child labor and for group activities, organized by a master gardener who guides the 
activities, without them ever undertake a programmatic form, as in schools. In the garden, the main activity is just the game, through which they develop the language, design and logical-mathematical activities.

The most typical example of the method as frobeliano continuity between play and learning is the pedagogy of "GIFTS" (ball, sphere, cube, cylinder, cube bricks).

Bruner says that the game is functional to the child as it allows him to experience problems, solutions and unrealistic behavior, thus facilitating creativity, experimentation and creativity. The game is therefore equivalent to a process of experience. Knowledge is in fact a process of categorization to Bruner, the tendency to simplify the many stimuli/external data by grouping into equivalence classes. More experience/knowledge brings me to have more than one category.

Cleparade, however, argues that we should not force the child in an activity unless the child does not feel a natural urge. All this implies a particular importance to the size of the effort, which is useful to overcome the obstacles that arise between him and the goal to achieve. The educator must arouse such an interest to ensure that the student is able to overcome the effort and this interest is only accessible through the use of joy and charm that only the game is able to provide. Education must therefore be joyful and playful.

Montessori says that the games are absolutely morally harmful as they lead to the dispersion of intelligence, which must instead be embodied in the child. His thinking is based on the total freedom of the student, as this only allows the development of spontaneous demonstrations, already present in the nature of the child.

Agazzi sisters argue that the child is a child who "does", both in the size of the game is that of practical life. They then organize specific environments for artistic production, mixing other activities on the aesthetic education (characterized by harmony and beauty) like drawing, free psychological expression of the child, or even a representation of a story educator.

Piaget, states that the purpose of education is to train the creative personality. That creativity is part of the game that plays a fundamental role in the development of intelligence in that it allows children to assimilate reality to the thought patterns of each. The game therefore represents a significant milestone in the overall development of the child's personality and should therefore be encouraged and valued.

\section{THE GAME AT SCHOOL}

The pedagogical freedom left to the teachers and the interest of the students have increasingly emerging pedagogy essentially geared towards the initiation sport.
When it comes to making a gesture finalized, a body technique effective, knowledge is no longer enough: We require those that are related to the processes of learning. The pedagogy is based on the tradition of evidence that should give a fair idea of the gesture to do so and cause a process of imitation. Are theories that animate the associazioniste learning pedagogical approaches. The gesture is broken down into the following elements to be acquired separately, then to coordinate and tie together. Gradually, this attitude analysis will give way to a more holistic approach, where the act of learning will be proposed as a whole to meet its original drive.

No longer are morphological or physiological differences that determine the working groups, but the level of performance achieved. From this point of view, the heterogeneity of the class requires individualization of teaching that take us away from the pedagogy of the formalism is identical for all year.

The development of the human sciences (particularly under the influence of genetic epistemology of Piaget), cognitive science and neuroscience and particularly of information theory, transform the pedagogical reflections. The demonstration assumes less importance, the student is led to enter more directly in the activity in order to identify its opportunities and its challenges. The teacher "system environment" playing on its transformations and the adjustment of the material in order to objectify the end of learning and of offering the maximum pupil information before, during and after the action, for which it evaluates the best procedures and results. Here you implemented a pedagogy of "problemsituations". The assessment procedures reveal the educational ambitions of teachers. In fact, when it comes to give a vote of physical education and sport in national examinations (in France), teachers consider that the only sports performance is insufficient to represent the progress and development of motor skills of the student. So they add an assessment of motor behavior generally defined as intelligence of movement, body awareness, mastery and effectiveness of the gesture. They also appreciated the knowledge about the activities followed. Under the conditions of teaching with times required, it is clear that there is a gap between intentions and reality.

\section{CONCLUSION}

To summarize the current pedagogical trend, we can consider that each sporting activity taught is proposed as a task or set of tasks to learn. These are referred to as 
elements that bring into play a set or a specific configuration of resources (biomechanics, bio-energy, bio-emotional, bio-information) that must later be able to mobilize for effective implementation and harmonic (ability). The bringing into play of resources involving the obligations of the environment and the characteristics of the task have the effect of enriching these resources considered necessary skills to be developed to allow the progressive complexification of specific expertise. These are special skills and general ability to inscribe in a program of physical education, from kindergarten to university. On the one hand it will give references to homogenize national sport on the organization of learning in school and on the other to define the cross-cutting objectives to develop, regardless of whether they are learning. The reflection teaching in the field of physical education and sports tends to find a way to tune the sociological need to preserve the diversity of learning sports-without falling into an accumulation of learning ever made-with the need to objectify acquisitions reinvesting outside of the activities pursued. Beyond the material conditions and hours of instruction as well as the methodological difficulties that can make illusory this ambition to produce a kind of "grammar of movement". We must not forget that every pedagogy is not easily pigeonholed in a formal program, when you know that the diversity of individual experiences in contexts extremely varied pupil is a singular being that it is to accompany us into a future. These brief notes pedagogical have shown that it has gone from a pedagogy of the collective preparatory trainer and a pedagogy of individual and team that puts the teacher in the face of social and individual meanings of the subject he teaches. In this sense we can understand that for many parents the professor of physical education and sport is a professor of gymnastics, for when the children became a professor of sports. You can see the proof that education is always at the service of a school culture that must continually redefine itself.

\section{REFERENCES}

Bertolini, P., 1996. Dictionary of Pedagogy and Educational Sciences. Freeman.

Cannao, M., 1989. Emotional problems in the education. School Publishing.

Castelnuovo, G., 2004. A. Ceriani-V. Colantonio, NLP training. De Vecchi Editore.

Contini, M.G., 1992. For a pedagogy of emotions. New Italy.

Demetrio, D., 1990. Professional educators. New Italy.

Demetrio, D., 1995. Education in adult life-for a phenomenological theory of the experiences and origins. NIS.

Demetrius, D., 1992. Micropedagogia, Qualitative research in education. New Italy.

Demetrius, D., 1996. Telling-the autobiography as selfcare. Routledge.

Dewey, J., 1974. Logic-Theory investigation. Einaudi.

Dewey, J., 1977. Human Nature and Conduct. New Italy.

Dewey, J., 1984. Experience and education. New Italy.

Frank, H., 1974. Pedagogy and Cybernetics. Armando.

Joints, A., 1983. Research and interdisciplinary work. The School Publishing.

Leo, L. and M. Prezza, 2003. Building and evaluating projects in the social. Franco Angeli.

Mattelart, A.M., 1997. History of the theories of communication.

Morselli, G., 1989. Dewey-Piaget-Husserl: A comparison. New Italy.

Mucchielli, R., 1987. Learn counseling. Centro Studi Erickson.

Pati, L., 1984. Pedagogy of educational communication. School Publishing.

Solomon, I., 1999. The pedagogical setting-constraints and opportunities for educational interaction. Carocci.

Zavalloni, 1979. Councillor pedagogical-The helping relationship in education. School Ed. 